\title{
Linear versus Nonlinear Filtering with Scale-Selective Corrections for Balanced Dynamics in a Simple Atmospheric Model
}

\author{
ANEESH C. SUbRAMANIAN \\ Scripps Institution of Oceanography, University of California, San Diego, San Diego, California \\ IBRAHIM HOTEIT \\ King Abdullah University of Science and Technology, Thuwal, Saudi Arabia \\ Bruce Cornuelle And Arthur J. Miller \\ Scripps Institution of Oceanography, University of California, San Diego, San Diego, California \\ HAJOON SONG \\ University of California, Santa Cruz, Santa Cruz, California
}

(Manuscript received 12 December 2011, in final form 11 April 2012)

\begin{abstract}
This paper investigates the role of the linear analysis step of the ensemble Kalman filters (EnKF) in disrupting the balanced dynamics in a simple atmospheric model and compares it to a fully nonlinear particle-based filter (PF). The filters have a very similar forecast step but the analysis step of the PF solves the full Bayesian filtering problem while the EnKF analysis only applies to Gaussian distributions. The EnKF is compared to two flavors of the particle filter with different sampling strategies, the sequential importance resampling filter (SIRF) and the sequential kernel resampling filter (SKRF). The model admits a chaotic vortical mode coupled to a comparatively fast gravity wave mode. It can also be configured either to evolve on a so-called slow manifold, where the fast motion is suppressed, or such that the fast-varying variables are diagnosed from the slow-varying variables as slaved modes. Identical twin experiments show that EnKF and PF capture the variables on the slow manifold well as the dynamics is very stable. PFs, especially the SKRF, capture slaved modes better than the EnKF, implying that a full Bayesian analysis estimates the nonlinear model variables better. The PFs perform significantly better in the fully coupled nonlinear model where fast and slow variables modulate each other. This suggests that the analysis step in the PFs maintains the balance in both variables much better than the EnKF. It is also shown that increasing the ensemble size generally improves the performance of the PFs but has less impact on the EnKF after a sufficient number of members have been used.
\end{abstract}

\section{Introduction}

The quality of the forecasts of the state of the atmosphere and ocean depends on the accuracy of the solutions of the partial differential equations (PDEs) describing these systems. Various data assimilation techniques have been developed over the last three decades to improve upon these solutions of the PDEs and to constrain the solutions to the ever-increasing number of observations.

Corresponding author address: Aneesh C. Subramanian, 9500 Gilman Dr., La Jolla, CA 92093-0224.

E-mail: acsubram@ucsd.edu
This helps in reducing the uncertainties in the forecast of these highly chaotic systems. The most popular data assimilation schemes in operational forecasts are the four-dimensional variational data assimilation (4DVAR) approach (Lewis and Derber 1985; Le Dimet and Talagrand 1986) and the ensemble Kalman filter (EnKF) approach (Evensen 1994; Tippett et al. 2003) and its variants (Ott et al. 2004; Mitchell and Houtekamer 2000; Song et al. 2010). More recently, approaches that are a hybrid of 4DVAR and EnKF (Hamill and Snyder 2000) are being tested in operational data assimilation systems to improve upon the errors of each method. 
The oceanic and atmospheric systems have multiple scales of dynamics interacting nonlinearly in time and space. For instance, the atmospheric system varies on time scales from climate to weather. The weather is fast varying, nonlinear, and chaotic and is known as an initial-value problem, whereas the climate is slowly varying and is known as a boundary value problem (Lorenz 1991). Ocean physics also involves multiple processes on multiple scales, from a few millimeters to thousands of kilometers in space and from seconds to decades in time. These are characterized by small-scale turbulent processes, mesoscale variability, and decadal and climate changes, all interacting with each other (Robinson and Lermusiaux 2004).

4DVAR and EnKF-based assimilation schemes have addressed the problem of forecasting multiscale systems to some extent. They have many associated problems with regard to the nonlinearity of the models and assimilating observations with dynamics at various time scales and measures of nonlinearity (Bennett 2002; Hoteit et al. 2005a; Van Leeuwen 2010). In meteorological and climatological applications, "balance" generally refers to the dominance of slow vortical motion over fast inertiagravity waves. In multiscale systems like the ocean or atmosphere in which modeled flows in certain regimes are expected to be balanced, it has been found that current 4D data assimilation techniques, as the EnKF and 4DVAR, could cause the excitation of spurious unbalanced motion (Polavarapu et al. 2000; Houtekamer and Mitchell 2005; Neef et al. 2006). This has been known to happen because of the development of unphysical correlations. Solutions to this problem have been attempted by imposing balance constraints on the analysis (Courtier and Talagrand 1990; Dee 1991; Todling and Cohn 1994; Polavarapu et al. 2000; Kepert 2004) or by filtering out fast modes. Imbalance in the flow due to the assimilated data cannot be completely corrected for even with such adjustments (Neef et al. 2009). Gershgorin and Majda (2010) show that a linear Kalman filter (KF) with model error performs better than an exact nonlinear extended Kalman filter (NEKF) for large-enough observation times in a nonlinear slow-fast system, yet they also show that the NEKF is stable for the case of strong fast forcing and hence for strong non-Gaussianity, unlike the linear KF. The application of the EnKF is still largely in a discussion/ testing phase (Lorenc 2003; Houtekamer and Mitchell 2005). The ability of this method to capture unbalanced motion is, however, still poorly understood (Neef et al. 2009). Szunyogh et al. (2005) is an example of a case in which an EnKF-type assimilation method was able to capture a fast-varying gravity wave mode that was present in observations and not in the model forecast, indicating that flow-dependent covariance models can potentially capture unbalanced motion. Neef et al. (2006) reported similar results where the EnKF was able to provide better estimates of the fast-varying modes than the linearized (extended) Kalman filter. Other techniques such as scaledependent modeling and assimilation using the ensemble Kalman filter have been attempted (Zou and Ghanem 2005) in engineering applications. This would be a more appropriate solution if the coupling between the scales were not highly nonlinear and chaotic as in the ocean or atmosphere dynamics.

4DVAR and the EnKF are equivalent when the system is Gaussian and linear (Kalnay et al. 2007), but both do not fully address the nonlinear estimation problem (Van Leeuwen 2010). The 4DVAR solution requires solving a nonconvex optimization problem while the EnKF analysis is still based on the linear correction step of the Kalman filter (Le Dimet and Talagrand 1986; Anderson 2001; Hoteit et al. 2008). For nonlinear data assimilation problems, the optimal solution is believed to be the solution of the Bayesian estimation problem, which involves the estimation of the conditional probability distribution function (PDF) of the system state given all available measurements up to the estimation time (Doucet et al. 2000). Knowledge of the state PDF allows for the determination of different estimates of the state, such as the minimum variance (MV) estimate (Todling 1999). The particle filter (PF) uses point-mass representation of the state PDF to provide a discrete approximation of the optimal nonlinear filter (ONF) (Doucet et al. 2000). In the PF, the particles are integrated forward with the numerical model to propagate the state PDF in time, and their assigned weights are updated every time new observations are available. The PF, however, suffers from the degeneracy phenomenon of its particles when most weights become concentrated on very few particles. Tackling this problem would require using resampling and a particle ensemble size sufficient to capture the effective state dimension (Snyder et al. 2008) of the assimilation system (Doucet et al. 2000). To alleviate this problem, Evensen (1994) proposed the EnKF while replacing the weight correction step by the linear Kalman correction step for each particle. This was shown to significantly enhance the robustness of the filter and allowed the implementation of the Bayesian filtering theory with small size ensembles of particles (Kivman 2003; Hoteit et al. 2008; Van Leeuwen 2010).

Despite recent successful implementations of the EnKF with various atmospheric and oceanic assimilation problems (Houtekamer and Mitchell 2005; Hoteit et al. 2005b; Pu and Hacker 2009), it is still not clear to what extent the linear correction step affects the accuracy of the filtering solution. Kivman (2003) compared the performances of the EnKF and the PF for the parameter estimation problem and found that the linear correction 
step of the EnKF fails to provide accurate estimates of the system parameters because of the strong nonlinear relation between the parameters and the observations. More recently, Jardak et al. (2010) compared the performances of the same filters in the presence of linear and nonlinear observation operators and concluded that the nonlinear filters are superior in the latter case. In this work, we are interested in assessing the impact of the linear correction step on the estimation of the different scales of the studied system. Following Neef et al. (2006), we address this problem using the simplified dynamical model of Lorenz (1986) (the model is hereafter called the Lorenz-86 model), which admits one nonlinear vortical mode and one inertia-gravity wave. We study the performance of the linear-based EnKF and two kinds of nonlinear filters, the sequential importance resampling filter (SIRF) and the sequential kernel resampling filter (SKRF). A similar problem has been considered by Neef et al. (2006), but studying the impact of linearization in the extended Kalman filter versus the ensemble approach, and by Neef et al. (2009), studying the impact of flow-dependent covariance evolution in the ensemble KF versus a static covariance model in an optimal interpolation (OI). In the Lorenz-86 model, we are mainly investigating whether the EnKF fails to capture the nonlinear coupling between the slow variable and the fast variable because of its linear correction step. This coupling between the slow and fast variables is preserved in the analysis state estimate of the PFs, and hence we show that they perform better in preserving the balance in the model as compared to the EnKF. When the dynamics lies on the slow manifold, we show that the EnKF performs as well as the PFs since the dynamics is approximately linear. We are particularly interested to see if the nonlinear correction step captures the fast-growing modes better compared to the EnKF and, if so, whether it still maintains the improvement in the analysis of the slowevolving mode. The second focus is to study the behavior of two different nonlinear filters compared to the EnKF for data assimilation in this model with varying observation frequencies and varying scales.

In the first set of experiments of this study we will evaluate the performance of the three different filters in the slow manifold framework of the Lorenz-86 model. The model is configured in the slow manifold with the fast modes set to zero and only the slowly varying vortical mode evolving in time. We test and show that the EnKF performs well compared to the two types of particle filters. In the second set of experiments we address the balanced flow problem, or flow where the vortical motion dominates and the inertial/gravitational motion is "slaved" to the dominant vortical mode flow. In the third set of experiments we will evaluate the accuracy of the three different filter analyses with a fully nonlinear model with the slowly varying vortical mode coupled to the fast inertia-gravity wave mode and each of them evolving in time independently.

The paper is organized as follows. The algorithms of the ensemble Kalman filter and the two types of particle filters are described and their characteristics compared in section 2. Section 3 describes the different configurations of the extended Lorenz- 86 model used in these experiments. The experimental setup describing the different model configurations and observation schemes used for experiments are presented in section 4. Section 5 contains discussion and conclusions.

\section{Linear and nonlinear Bayesian filtering}

The Bayesian filtering approach is presented first and then approximations for its Monte Carlo implementation are presented in the forms of the PF and the EnKF.

To describe the Bayesian filtering algorithm, consider the following nonlinear stochastic discrete-time dynamical system:

$$
\begin{aligned}
& \mathbf{x}_{k}=\mathbf{M}_{k}\left(\mathbf{x}_{k-1}\right)+\eta_{k}, \\
& \mathbf{y}_{k}=\mathbf{H}_{k}\left(\mathbf{x}_{k}\right)+\epsilon_{k},
\end{aligned}
$$

where $\mathbf{x}_{k}$ is the state vector (to be estimated), of dimension $n ; \mathbf{y}_{k}$ is the observation vector, of dimension $p$; $\mathbf{M}_{k}$ and $\mathbf{H}_{k}$ are two continuously differentiable maps from $\mathbb{R}^{n}$ to $\mathbb{R}^{n}$ and from $\mathbb{R}^{n}$ to $\mathbb{R}^{p}$, respectively representing the transition and the observational operators; and $\eta_{k}$ and $\epsilon_{k}$ denote the dynamical and the observational noise, respectively. The conditional distributions of $\eta_{k}$ and $\epsilon_{k}$, given $\mathbf{x}_{0}, \ldots, \mathbf{x}_{k}$, are assumed to be Gaussian with zero mean and nonsingular covariance matrices $\mathbf{Q}_{k}$ and $\mathbf{R}_{k}$, respectively.

Starting from a random initial condition with a known probability density function, the Bayesian filter provides the conditional density function of the system state given all available measurements up to the estimation time. To simplify the notation, we define $\mathbf{y}_{1: k}$ as a shorthand for the set of observations $\mathbf{y}_{1}, \ldots, \mathbf{y}_{k}$ previous to time $t_{k}$. Let $p_{k}^{f}\left(\cdot \mid \mathbf{y}_{1: k-1}\right)$ be the conditional (predictive) density of $\mathbf{x}_{k}$ given $\mathbf{y}_{1: k-1}$ and $p_{k}^{a}\left(\cdot \mid \mathbf{y}_{1: k}\right)$ be the conditional (analysis) density of $\mathbf{x}_{k}$ given $\mathbf{y}_{1: k}$, both determined at time $t_{k}$. The Bayesian filter recursively operates with a succession of prediction and correction steps as summarized below. The reader is referred to Doucet et al. (2000) for an extensive description of the filter.

- Prediction step: The predictive density $p_{k}^{f}\left(\cdot \mid \mathbf{y}_{1: k-1}\right)$ is obtained by integrating $p_{k-1}^{a}\left(\cdot \mid \mathbf{y}_{1: k-1}\right)$ with the model 
(1) to the time of the next available observation $t_{k}$. The conditional density of the state vector $\mathbf{x}_{k}$ to be at $\mathbf{x}$ at time $t_{k}$ given that it was at $u$ at time $t_{k-1}$ is $\phi\left[\mathbf{x}-\mathbf{M}_{k}(\mathbf{u})\right.$; $\left.\mathbf{Q}_{k}\right]$, where

$$
\phi(\mathbf{x} ; \mathbf{\Sigma})=\frac{1}{\sqrt{\operatorname{det}(2 \pi \mathbf{\Sigma})}} \exp \left(-\frac{\mathbf{x}^{\mathrm{T}} \mathbf{\Sigma}^{-1} \mathbf{x}}{2}\right)
$$

denotes the Gaussian density of zero mean and covariance matrix $\mathbf{\Sigma}$. It is emphasized that this forecast procedure is valid for discrete time formulations. Thus,

$$
p_{k}^{f}\left(\mathbf{x} \mid \mathbf{y}_{1: k-1}\right) \int_{\mathbb{R}^{n}} \phi\left[\mathbf{x}-\mathbf{M}_{k}(\mathbf{u}) ; \mathbf{Q}_{k}\right] p_{k-1}^{a}\left(\mathbf{u} \mid \mathbf{y}_{1: k-1}\right) d \mathbf{u} .
$$

- Correction step: After a new observation $\mathbf{y}_{k}$ is made, the analysis density $p_{k}\left(\cdot \mid \mathbf{y}_{1: k}\right)$ at time $t_{k}$ is determined by "correcting" the predictive density with the new observation using the Bayes rule,

$p_{k}^{f}\left(\mathbf{x} \mid \mathbf{y}_{1: k-1}\right)=\frac{1}{b_{k}} p_{k}^{f}\left(\mathbf{x} \mid \mathbf{y}_{1: k-1}\right) \phi\left[\mathbf{y}_{k}-\mathbf{H}_{k}(\boldsymbol{x}) ; \mathbf{R}_{k}\right]$.

The analysis density is therefore obtained by multiplying the prior predictive density by the observation density and normalizing by $b_{k}=\int_{\mathbb{R}^{n}} p_{k}^{f}\left(\mathbf{u} \mid \mathbf{y}_{1: k-1}\right) \phi\left[\mathbf{y}_{k}-\right.$ $\left.\mathbf{H}_{k}(\mathbf{u}) ; \mathbf{R}_{k}\right] d \mathbf{u}$ to ensure a probability density.

While the expressions of the state PDF can be easily obtained, determining the value of the predictive density at each point of the state space is practically impossible for large dimensional systems (Doucet et al. 2000). This actually requires the evaluation of the model $\mathbf{M}_{k}(\mathbf{x})$ for a prohibitive number of values of $\mathbf{x}$, knowing that even one single evaluation can be computationally prohibitive in realistic atmospheric and oceanic applications (Snyder et al. 2008). The particle filter and the ensemble Kalman filter are two discrete approximations of the Bayesian filter. Here we describe the characteristics of these two Monte Carlo implementations of the Bayesian filter.

\section{a. The particle filter}

The PF provides a discrete solution of the Bayesian filtering problem using point-mass representations $\sum_{i=1}^{N} w_{i} \delta_{\mathbf{x}^{i}}$, of the state PDFs (Doucet et al. 2000). The vectors $\mathbf{x}^{i}$ are called particles and the $w_{i}$ are their associated weights; $N$ is the number of particles (or the size of the ensemble). After a forecast or analysis step, the minimum variance estimate of the system state is then obtained as the weighted average of the ensemble
$\sum_{i=1}^{N} w^{i} \mathbf{x}^{i}$. Starting from an initial ensemble of particles $\mathbf{x}_{0}^{i}, i=1, \ldots, N$, the PF algorithm consists of a prediction step to integrate the particles in time and a correction step to update the weights as follows:

- Prediction step: At time $t_{k-1}$, the particles $\mathbf{x}_{k-1}^{i}$ are integrated forward with the model to the time of the next available observation $t_{k}$.

- Correction step: The new observation is used to update the weights with

$$
w_{k}^{i}=\frac{1}{c_{k}} w_{k-1}^{i} \phi\left[\mathbf{y}_{k}-\mathbf{H}_{k}\left(\mathbf{x}_{k}^{i}\right) ; \mathbf{R}_{k}\right]
$$

where $c_{k}$ is a constant normalizing the total weight. The particles remain unchanged. Thus a particle receives more or less weight proportional to its distance from the most recent observation normalized by the observational error covariance matrix $\mathbf{R}_{k}$.

In practice, the PF suffers from a major problem known as the degeneracy phenomenon (Doucet et al. 2000); after several iterations most weights become concentrated on very few particles. This happens because the particles drift away from the true state with the observations exerting no feedback on the particles. The "effective" size of the ensemble as described by Snyder et al. (2008) decreases over time and after a few assimilation steps only a small fraction of the ensemble contributes to the filter solution, causing very often the divergence of the filter. Resampling was introduced as a way to get around this problem. This Monte Carlo technique basically consists of drawing new particles according to the PDF of the ensemble and then reassigning them the same weights (Doucet et al. 2000). In most applications, the new particles are drawn from the discrete approximation of the state PDF. This is known as SIRF. To avoid drawing similar particles when the system noise is absent or insignificant, the particles might be also resampled from an approximating continuous PDF (Pham 2001). This is known as SKRF. Moreover, even with resampling, the PF would still require a large number of particles to provide an accurate solution (Doucet et al. 2000). This makes brute-force implementation of the PF with computationally demanding atmospheric and oceanic models quite a challenging problem (Anderson 2003; Snyder et al. 2008; Van Leeuwen 2009).

\section{b. Ensemble Kalman filtering}

To avoid the problems associated with the application of the PF and the KF with large dimensional nonlinear problems, (Evensen 1994) introduced the EnKF as a hybrid approach between the KF and the PF. The basic idea behind this filter is to combine the optimal forecast 
step of the PF with a KF correction of the particles. The weights are then kept uniform. The algorithm of the EnKF can be summarized as follows:

- Prediction step: As in the PF, the analyzed particles $\mathbf{x}_{k-1}^{a, i}$ are advanced in time with the model to compute the forecast particles $\mathbf{x}_{k}^{f, i}$.

- Correction step: A KF correction step is applied to every forecast particle as

$$
\mathbf{x}_{k}^{a, i}=\mathbf{x}_{k}^{f, i}+\mathbf{G}_{k}^{e}\left[\mathbf{y}_{k}^{i}-\mathbf{H}_{k}\left(\mathbf{x}_{k}^{f, i}\right)\right]
$$

The gain matrix $\mathbf{G}_{k}^{e}$ is the same as the Kalman gain but is computed from the sample covariance matrix of $\mathbf{x}_{k}^{f, i}$ as described by Evensen (2003).

In (7), the observation was assigned a superscript index associated with each particle. In contrast to the deterministic alternatives known as the ensemble square root filters (Tippett et al. 2003), which do not require perturbing the observations for the analysis computations, the observation needs to be perturbed by noise sampled from the PDF of the observational error (Burgers et al. 1998) for the EnKF.

The correction step of the EnKF uses only the first two moments of the particles ensemble and is thus suboptimal for non-Gaussian systems. In practical situations, however, the EnKF was found to be more robust than the PF when small-size ensembles were used because the Kalman update of the particles is applied using the forecast error covariance matrices estimated from the particles ensemble. This made the implementation of the Bayesian filtering feasible with high-dimensional systems, as in meteorology and oceanography. The KF correction reduces the collapse of the ensemble by "pulling" the particles toward the true state of the system, allowing the filter to operate with a reasonable number of particles (Kivman 2003; Hoteit et al. 2008; Van Leeuwen 2009). With large enough ensembles, however, the PF was shown to outperform the EnKF (Nakano et al. 2007; Jardak et al. 2010). It is still an active area of research as to the defects of a Kalman filter-type linear correction that can be overcome by using the fully nonlinear (non-Gaussian) Bayesian correction in filtering highly nonlinear systems such as the ocean or the atmosphere.

\section{Lorenz-86 model description}

\section{a. Lorenz-86 model}

The model used in this study is that of Lorenz (1986), as modified by Wirosoetisno and Shepherd (2000, hereafter WS00), and will be referred to here as the extended Lorenz model or exL86. It has only 4 degrees of freedom but admits both a fast gravity wave and a chaotic vortical mode, with an asymptotic, nonlinear balance between fast and slow variables. The advantage of models such as exL86 is that the balance between fast and slow variables is well understood, and the assimilated analysis can thus be easily interpreted in terms of the balanced and unbalanced components of the motion. The fact that this model is conservative does not pose a great difficulty, since the intention here is to use it to study assimilation algorithms in the context of the slow versus the fast variables. As pointed out by Lorenz (1986) and WS00, dissipation of gravity waves is not the cause of the existence of a slow manifold, and therefore models such as this one can be quite representative of realistic balanced dynamics.

The basic equations of the model are as follows:

$$
\begin{aligned}
\frac{d \phi}{d t} & =w, \\
\frac{d w}{d t} & =-\frac{C}{2} \sin (2 \phi+2 \epsilon b x), \\
\frac{d x}{d t} & =-\frac{z}{\epsilon}, \\
\frac{d z}{d t} & =\frac{x}{\epsilon}+\frac{b C}{2} \sin (2 \phi+2 \epsilon b x),
\end{aligned}
$$

where $C=1+0.8 \cos (0.92 t)$, with $t$ as time; $\epsilon=0.1$ and $b=0.71$ are two other constants.

Equations (8)-(11) describe a chaotic vortical mode in $\phi$ and $w$, coupled to a linear gravity wave in $x$ and $z$. The four variables are the spectral coefficients of potential vorticity $\phi$ and $w$ (note that $\phi$ is actually related to the phase of two potential vorticity coefficients from the original derivation; Lorenz 1986), geostrophic imbalance $z$, and divergence $x$. The parameter $b$, which couples the fast and slow normal modes, corresponds to the rotational Froude number and the parameter $\epsilon$ is related to the parameter $b$ and the Rossby number by the following equation:

$$
\epsilon \equiv \frac{\operatorname{Ro} B}{\sqrt{1+B^{2}}},
$$

where Ro $=U / f L$ is the Rossby number and $B=f L / \sqrt{g H}$ is the rotational Froude number. Ro is the ratio of the inertial time scale to the advective time scale of the flow, and $B$ is ratio of the Coriolis force to the gravitational restoring force. The value of these parameters indicates a time scale of separation between the different normal modes (Saujani and Shepherd 2006). The model can be run in various configurations to mimic the dynamics of coupled and uncoupled scales of motion with only the 
slowly varying mode active or in a slaved configuration with the fast varying mode slaved to the slow manifold.

\section{b. Slow manifold initialization}

The model can be configured in a slow manifold such that two variables vary at a slow time scale and the fast varying variables are set to be zero. The lowest-order approximation to a slow manifold in the exL86 system is found by setting $x=z=0$ and evolving only $\phi$ and $w$. For $\epsilon=0$ or $b=0$, this manifold is exact, and results in the single-time scale system

$$
\begin{aligned}
& \frac{d \phi}{d t}=w, \\
& \frac{d w}{d t}=-\frac{C}{2} \sin (2 \phi) .
\end{aligned}
$$

This system is analogous to a chaotic pendulum when $C$ is still time dependent in this configuration. This corresponds physically to the quasigeostrophic equations as in both systems the fast gravity waves are filtered out. To establish how nonlinearity of the slow mode affects data assimilation, experiments with this model are performed using the ensemble Kalman filter and the two flavors of the nonlinear particle filter. Results are presented in section 5 .

\section{c. Second-order slaving relations}

The model can also be configured in a slaved relations mode such that the fast varying modes are only dependent on the slow variables and are time independent:

$$
\begin{aligned}
& x=-\frac{\epsilon}{2} C b \sin 2 \phi+O\left(\epsilon^{3}\right), \\
& z=\epsilon^{2}\left[C b w \cos 2 \phi+\frac{C^{\prime}}{2} b \sin (2 \phi)\right]+O\left(\epsilon^{3}\right),
\end{aligned}
$$

where $C^{\prime}$ is the time derivative of $C$.

To assess the efficiency of the nonlinear analysis step in enhancing the dynamical balance of the filter solutions, identical twin assimilation experiments were designed such that the true state was balanced, but the observational errors were projected onto all degrees of freedom, including the fast modes. Results are presented in section 5 .

\section{Experiments setup and objectives}

We set up experiments to test the performance of the EnKF compared to the two flavors of particle filters, the SIRF and the SKRF. The ExL86 model is configured in three different modes. The first configuration used is the slow manifold configuration of the model in which the fast variables were set to zero and only the slow variables were allowed to evolve in time. This model configuration is weakly nonlinear in its dynamics.

The second configuration considered is the slaved mode with the fast variables depending completely on the evolution of the slow variables and not evolving in time independently. This configuration of the model has the slow variable evolving in time uncoupled to the fast variable. Filtering on this configuration will help us understand how the linear versus the nonlinear filter improves the slow mode and hence also influences the evolution of the fast mode without coupling back to the slow mode.

The third configuration is the fully nonlinear mode with the slow and the fast variables coupled and evolving in time separately as the full dynamics dictates. This model configuration enables us to assess whether the filters are improving the fully nonlinear coupled dynamics of the model or only the slow or fast modes individually. This would give us an insight into how important it is to have a linear versus a nonlinear correction step to capture the dynamics of a fully nonlinear coupled model.

In all experiments presented here, we chose a trajectory of the model starting from the same initial conditions of $\phi=-6.617, w=-0.449, x=0$, and $z=0$. In all these three configurations with three different types of filters, we investigated two different sets of observation schemes. One observation scheme was to observe all variables at all time. This observation scheme is called the all observation experiment. The second observation scheme was to observe only the first (slow) variable and the third (mixed) variable every eight time steps. This observation scheme is referred to as the sparse observation experiment. We also investigated assimilating observations from only the slow variable. We noticed that the errors are higher than in the sparse observations experiment but do not present the results from these experiments for the sake of brevity. All assimilation experiments were done with 10 and 25 ensemble members. Results using the 10 member ensemble (not shown here) depict an overall weaker performance for the particle filters compared to the 25-member ensemble case. Results from the experiments with 25 ensemble members are presented in this study for the different filter solutions. An inflation factor of 1.01 was used for the EnKF simulations to account for subsampling errors in the EnKF (Hamill et al. 2001). The model time step was chosen to be 0.01 and the observational error variance used was a percentage of the total variance of each variable derived from the climatology of the system.

\section{Results from experiments}

Results from the experiments with the three separate modes of the model are presented in this section. 

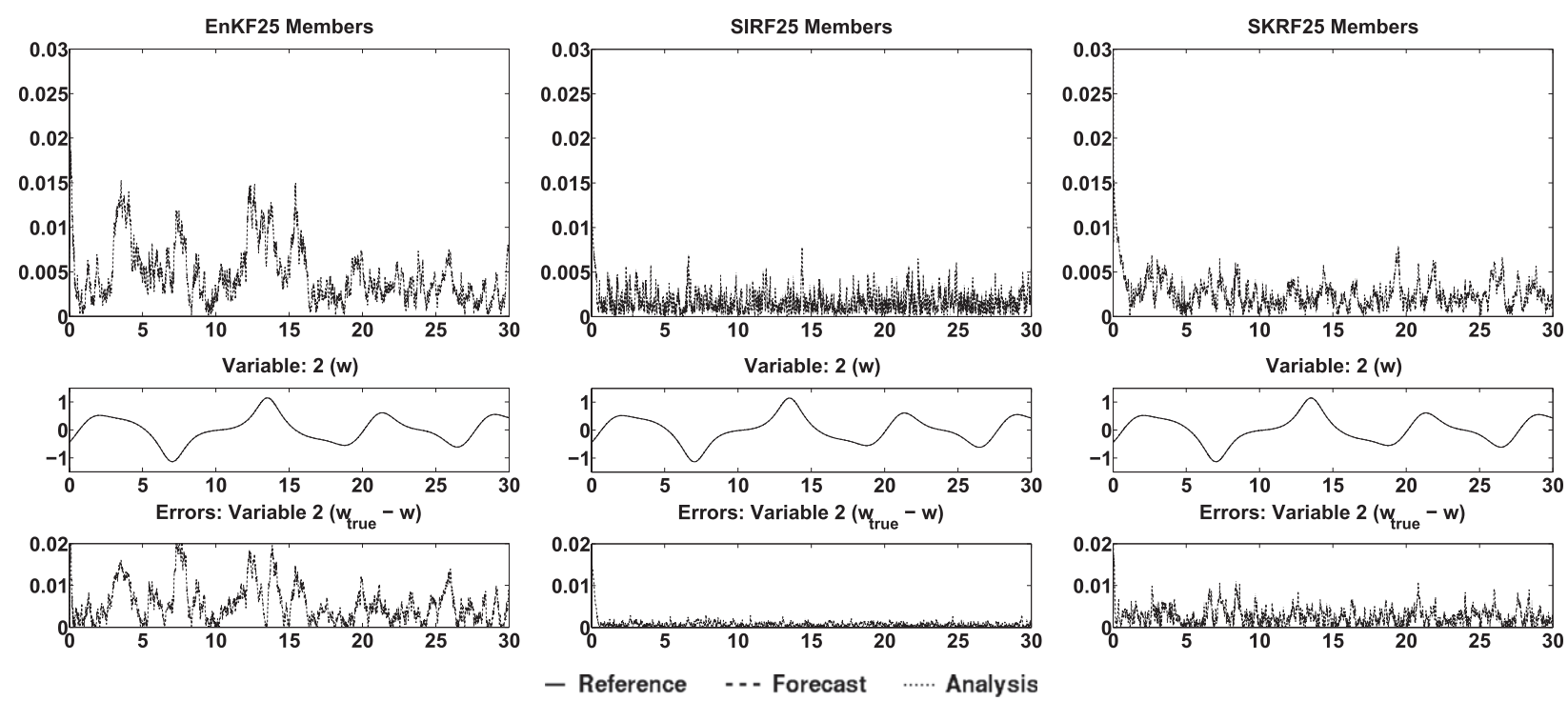

FIG. 1. Slow manifold: Every variable at all time steps is observed. The system is known perfectly. It can be observed that both EnKF and PFs capture the slow manifold well when all variables are observed.

\section{a. Slow manifold}

To separate the problem of balance from that of general nonlinearity and chaos, we establish how well the EnKF, SIRF, and SKRF estimate the model state in the single-time-scale slow manifold configuration of the model. In the slow manifold case, only the variables $x$ and $z$ are varying in time and variables $\phi$ and $w$ are zero at all time. When all variables at all times are observed in the assimilation experiments, the EnKF estimates the slow variable with low error comparable to that of the nonlinear filters' solutions as shown in Fig. 1. This shows that when the model has only a slowly varying single time scale that is weakly nonlinear, the nonlinear filters are comparable in estimating the state to the EnKF, which is based on Gaussianity assumptions. Similarly, in Fig. 2, we see the solutions from the three assimilation schemes for the sparse observation experiment. This experiment shows that the SKRF estimates the variable $w$ with lesser RMSE than SIRF and is comparable to the
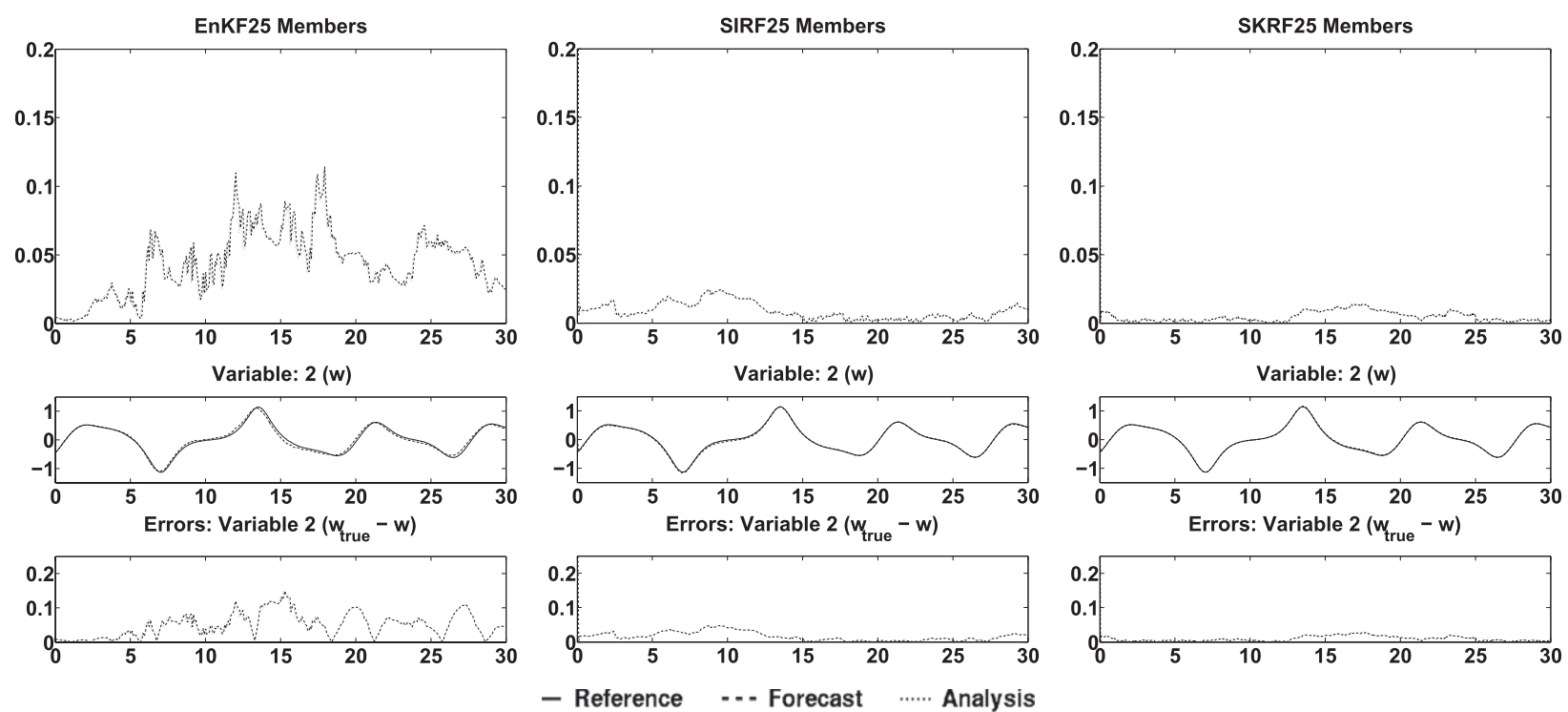

- Reference -..- Forecast …... Analysis

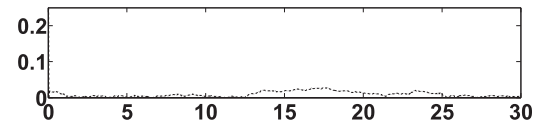

FIG. 2. Slow manifold: First and third variables at every eighth time step are observed. Here we observe that the system is not well captured by the EnKF analysis whereas the PFs capture the system well. 

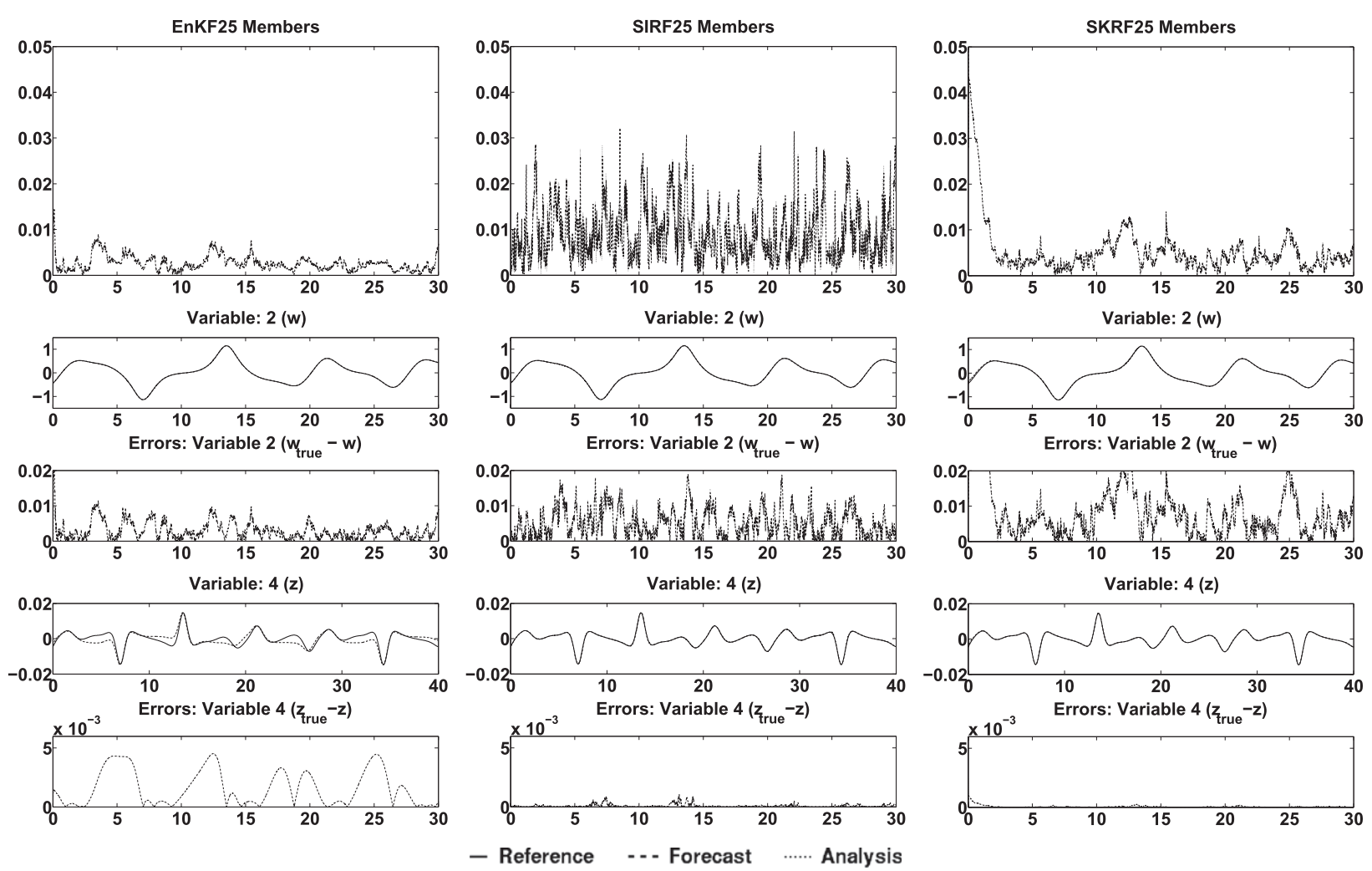

FIG. 3. Slaved manifold: Every variable at all time steps is observed. The system is known perfectly. It can be observed that both EnKF and PFs capture the slow manifold well when all variables are observed.

EnKF RMSE (after a few time steps of assimilation). The advantage of the PFs is that the balance of the dynamic variables relies only on a balanced forecast ensemble that is not changed by a linear correction step such as in an ensemble Kalman filter. Neef et al. (2006) show that a more balanced error covariance in an EnKF is the reason it captures the dynamics better than an extended Kalman filter (EKF). They also argue that overobserving can lead to a more unbalanced forecast ensemble and thus to an unbalanced analysis, yet in our experiments reduced observations lead to higher errors in all three filters, more so in the EnKF.

\section{b. Second-order slaving relations}

In Fig. 3, the model was configured as an approximation to the slow manifold, where the evolution of the system depends on the slow variables only. The fast variables are found diagnostically as functions of the slow variables, and the gravity wave is suppressed. It can be seen that the EnKF estimate does not capture the transition between the peaks in the variable $z$. The reason for this failure could be that the EnKF updates the ensemble trajectories using a linear analysis estimate and hence could destroy the nonlinear interactions between the variables, while the SIRF and SKRF only change the ensemble probabilities but do not modify their trajectory. Hence, the nonlinear filters could retain the balance relationship to the extent that ensemble members themselves are balanced and the forecasts in the ensemble use the full nonlinear model and the gravity wave is therefore bounded. The RMSE for variable $z$ (fast variable) is lowest for the SKRF. The peaks in variable $z$ are captured well by both the SKRF and SIRF estimates. The total RMSE is lowest for SKRF.

Figure 4 shows the model estimates for the same slaved mode configuration with sparse observations. Again, SKRF state estimates have the lowest RMSE compared to the SIRF and EnKF state estimates. As in Fig. 3, the EnKF state estimate is unable to infer the transition between peaks in the variable $z$, which is inferred better in the SIRF and SKRF solutions. Although variable $w$ is estimated well by all three filters, the SKRF performs better with this variable. Hence, the nonlinear filter, especially with particles sampled from a continuous PDF, estimates the solutions to the highly nonlinear model with scale separation better in both slow and fast time scales. The balance relationship between the slow modes and fast modes is simulated better by the nonlinear filters 

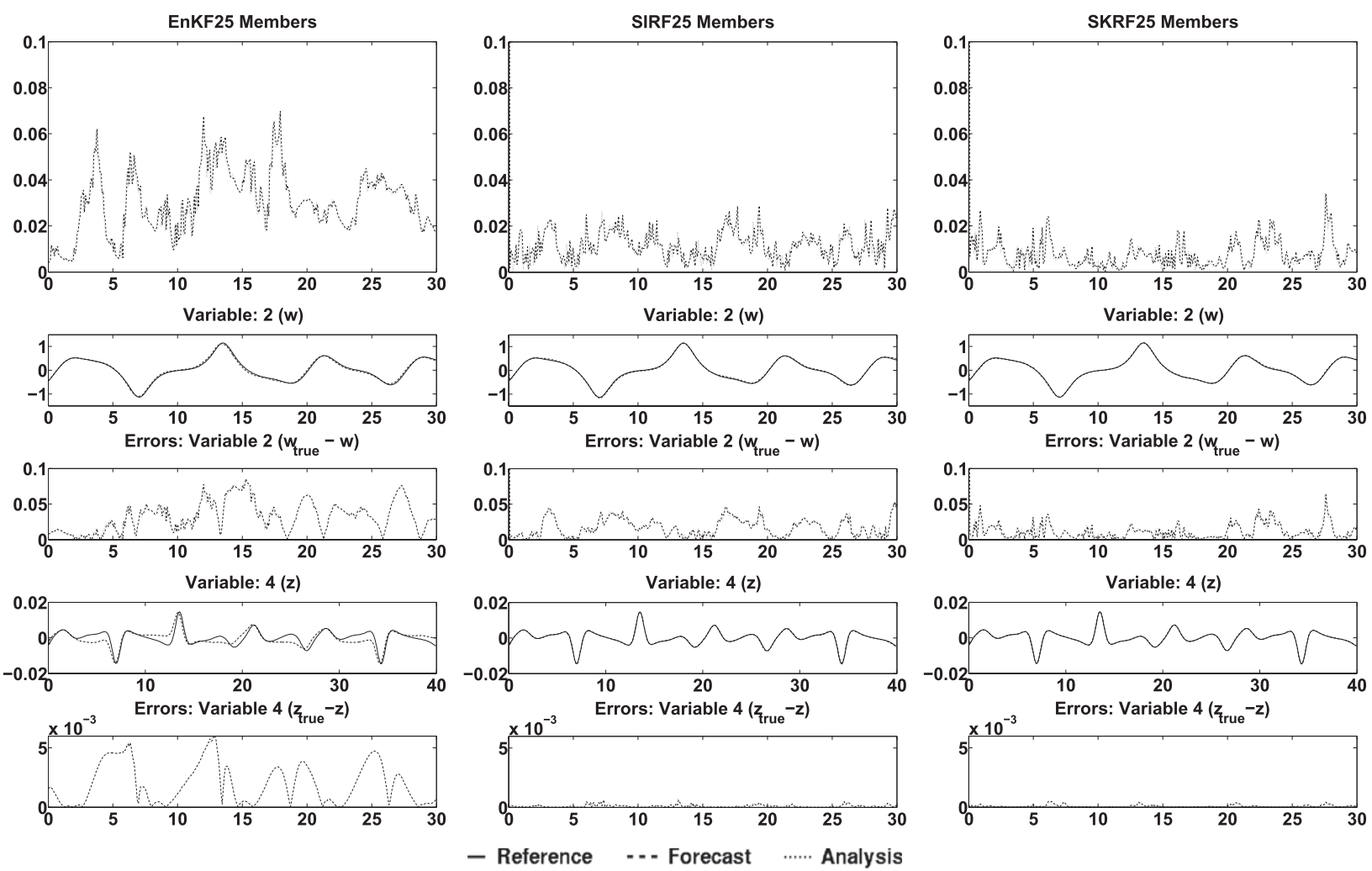

FIG. 4. Slaved manifold: First and third variables at every eighth time step are observed. Here we observe that the system is not well captured by the EnKF analysis whereas the PFs capture the system well.

with better estimated fast-slow statistics used to update the fast variables with observations of the slow variables. This is also in agreement with the results of Neef et al. (2009), where it is shown that an estimate of the fast variables from observations of a slow variable alone requires the fast-slow error covariances to capture the balance relationship.

\section{c. Nonlinear mode}

Figure 5 shows the total RMSE and the RMSE for variables $w$ and $z$. The RMSE for all three filters, EnKF, SIRF, and SKRF, are comparable and low. This shows that when assimilating "frequent observations" (i.e., in time periods much shorter than the error doubling time) of all variables in a nonlinear model with interacting slow modes and fast modes, all three filters capture the variables well and maintain the balance among the variables. The slow mode is captured better by the EnKF than the fast mode as seen in Fig. 6, where we assimilate sparse observations. The EnKF state estimate of the unobserved slow mode variable $w$ and fast variable $z$ are worse than the estimates by the nonlinear filters SIRF and SKRF. The nonlinear filters are comparable to each other and perform well in estimating both the slow and fast variables.
The transition between peaks in the fast variable are still not well captured in these two schemes, yet they do capture them well in time as compared to the EnKF solutions. This is consistent with results from Neef et al. (2006), where it is shown that a nonlinear evolution in the model such as in PFs slows the growth in imbalance in the model analysis and also allows for efficient assimilation of sparser observations without a great loss of balance. Neef et al. (2006) also show that the ensemble averaging in the EnKF helps in keeping a balanced state over an extended Kalman filter-like approach. This advantage is even better preserved in the weighted mean of the PFs.

We have also tested this assimilation experiment with various ensemble sizes from 10 to 100 and have shown the total RMSE for each variable in each of the ensemble size cases in Fig. 7. This figure shows that, for the EnKF, increasing the ensemble size from 10 to 100 does not significantly change the skill of the filter. For the PFs, increasing the ensemble size from 10 to 50 improves the skill of the filters but further increase in the ensemble size does not gain much in terms of reducing the RMSE. Even an ensemble size of 50 is much above the dimension of the problem being solved. Yet to model the PDFs of 

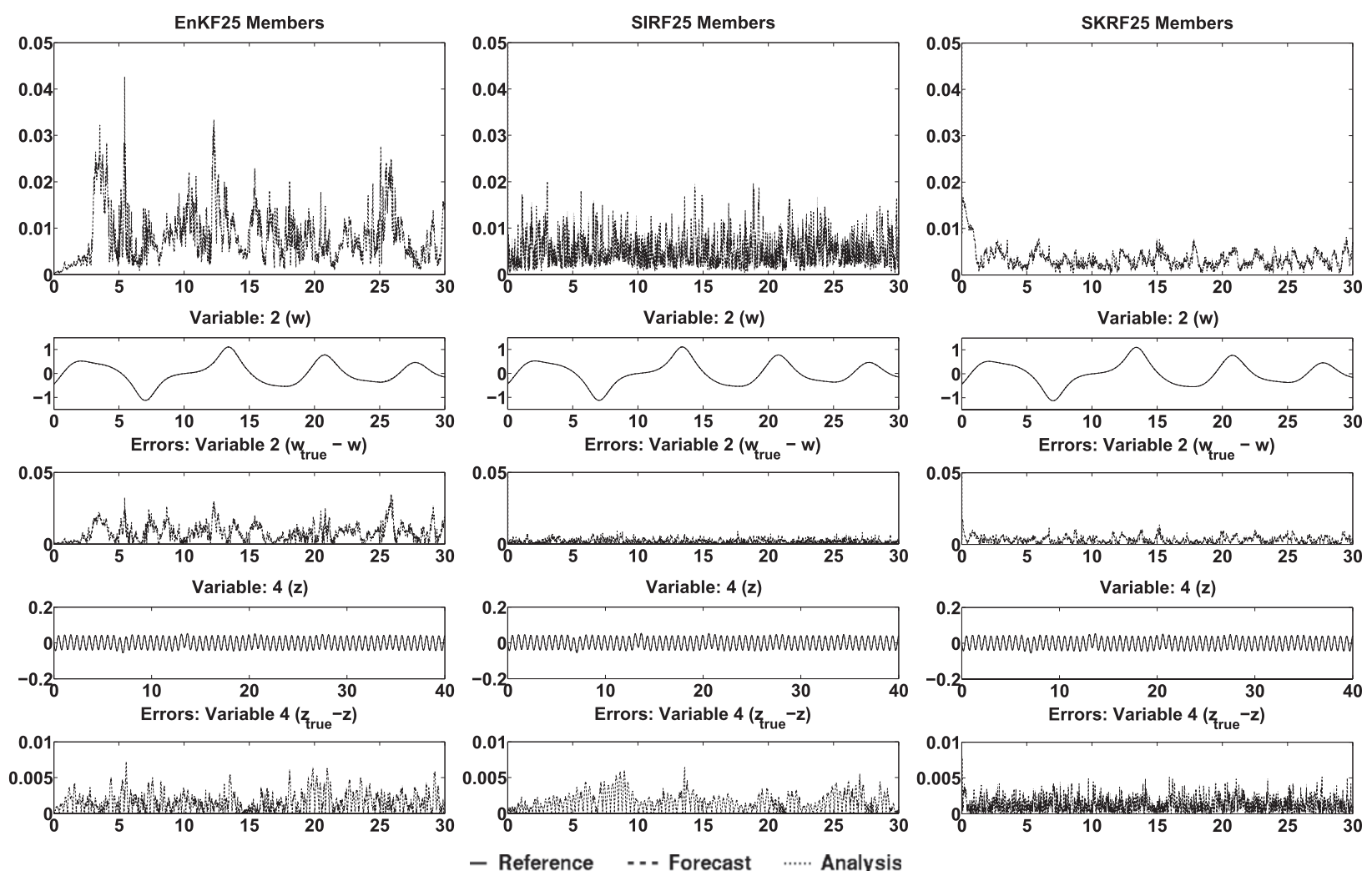

FIG. 5. All modes: Every variable at all time steps is observed. The system is known perfectly. It can be observed that both EnKF and PFs capture the slow manifold well when all variables are observed.

each variable appropriately and integrate it forward in time, it is necessary to sample the variable PDFs sufficiently in order to capture the most information.

One of the main properties of this model is the nonlinearity in the interaction of different modes and the non-Gaussianity of the fast mode. Both the nonlinear coupling and the fast forcing imply non-Gaussian PDFs for the fast variable. Hence, the model was run for all three filters with 1000 ensemble members so as to get a good estimate of the prior and posterior estimate for the PDFs of the model variables for the fully nonlinear configuration. The PDFs of the state are estimated using a Gaussian mixture model (GMM), a parametric probability density function represented as a weighted sum of Gaussian component densities (McLachlan and Peel 2000; Anderson and Moore 1979).

A Gaussian mixture model is a weighted sum of $N$ Gaussian densities:

$$
p(x \mid \sigma)=\sum_{i=1}^{N} w_{i} g\left(x \mid \mu_{i}, \Sigma_{i}\right)
$$

where $x$ is the measured quantity, $w_{i}$ are the Gaussian mixture weights, and $g\left(x \mid \mu_{i}, \Sigma_{i}\right)$ are the component
Gaussian probability density functions. The mixture weights satisfy the constraint that $\sum_{i=1}^{N} w_{i}=1$. The collective set of parameters is represented as

$$
\sigma=\left\{w_{i}, \mu_{i}, \Sigma_{i}\right\}, \quad i=1, \ldots, N .
$$

Plotting the PDFs of the variables shows the nonGaussian nature of the PDFs of the variables in the fully nonlinear case. Doucet et al. (2000) show theoretically that the estimated PDF from the PFs converge to the Bayesian PDF as the number of samples tends to infinity. The EnKF always approximated the PDF to be Gaussian but the particle filters are able to capture a non-Gaussian PDF and evolve it forward in time. This could be a critical reason for the PFs to perform better than the EnKF.

Figure 8 shows that in the forecast and the analysis, the particle filters capture the non-Gaussian PDFs in their ensemble spread. When we reduce the number of observations in time, both the forecast and analysis PDFs conditioned on the observations increase in their spread, yet the PFs do have a non-Gaussian PDF that is likely to represent the non-Gaussianity of the system better, as shown in Fig. 9. 

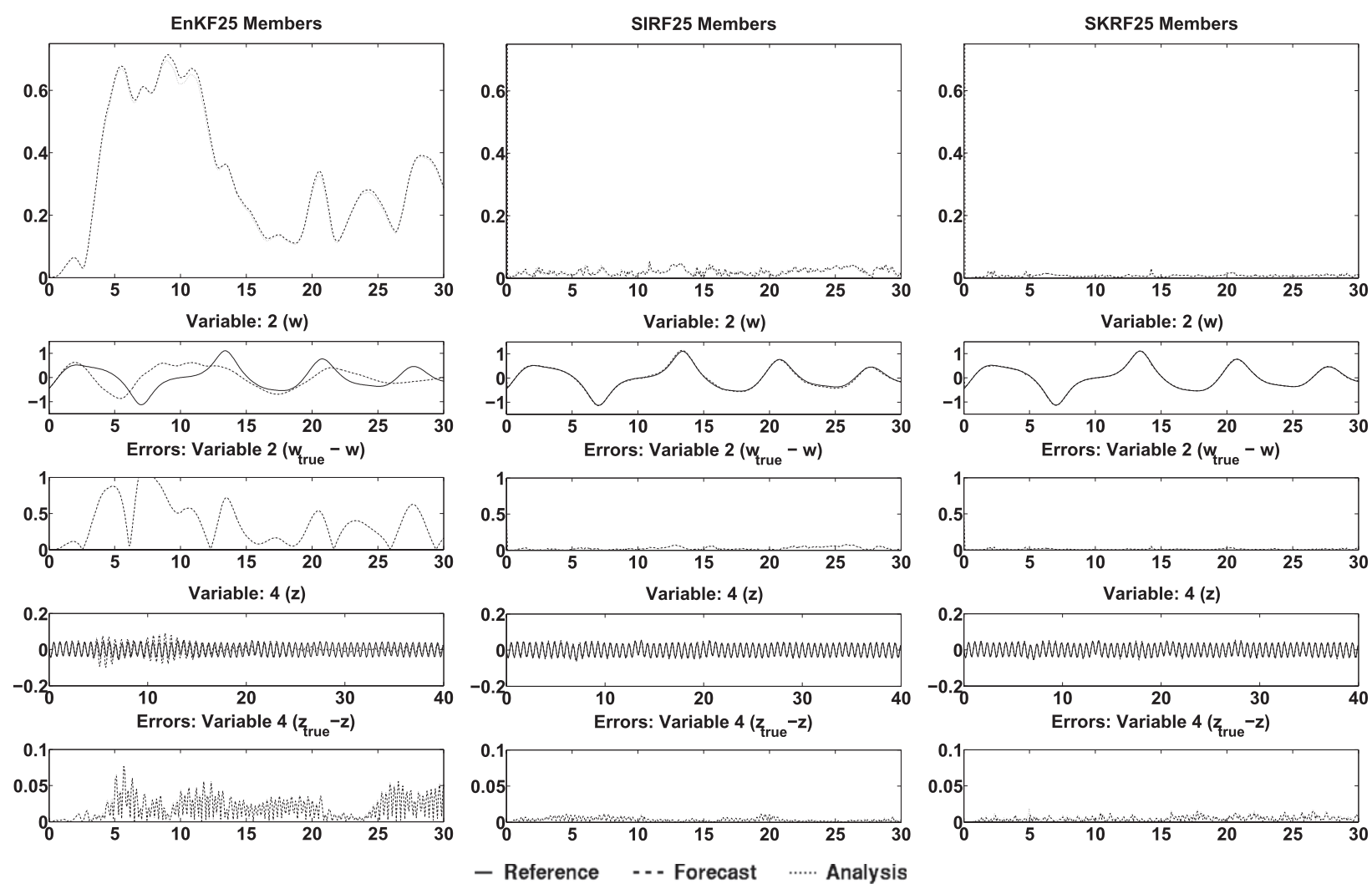

FIG. 6. All modes: First and third variables at every eighth time step are observed. Here we observe that the system is not well captured by the EnKF analysis whereas the PFs capture the system well.

Neef et al. (2009) show that the EnKF analysis cycle can cause the ensemble to lock onto a gravity wave of the wrong amplitude, causing filter divergence in the analysis of the fast mode, even in regimes where it converges for the slow mode. Fast-mode filter divergence comes about because the linear gravity wave ensemble does not spread between observations. This drawback is not overcome even when observations are very frequent. In realistic applications it is possible that gravity waves that are present in the truth may not be represented
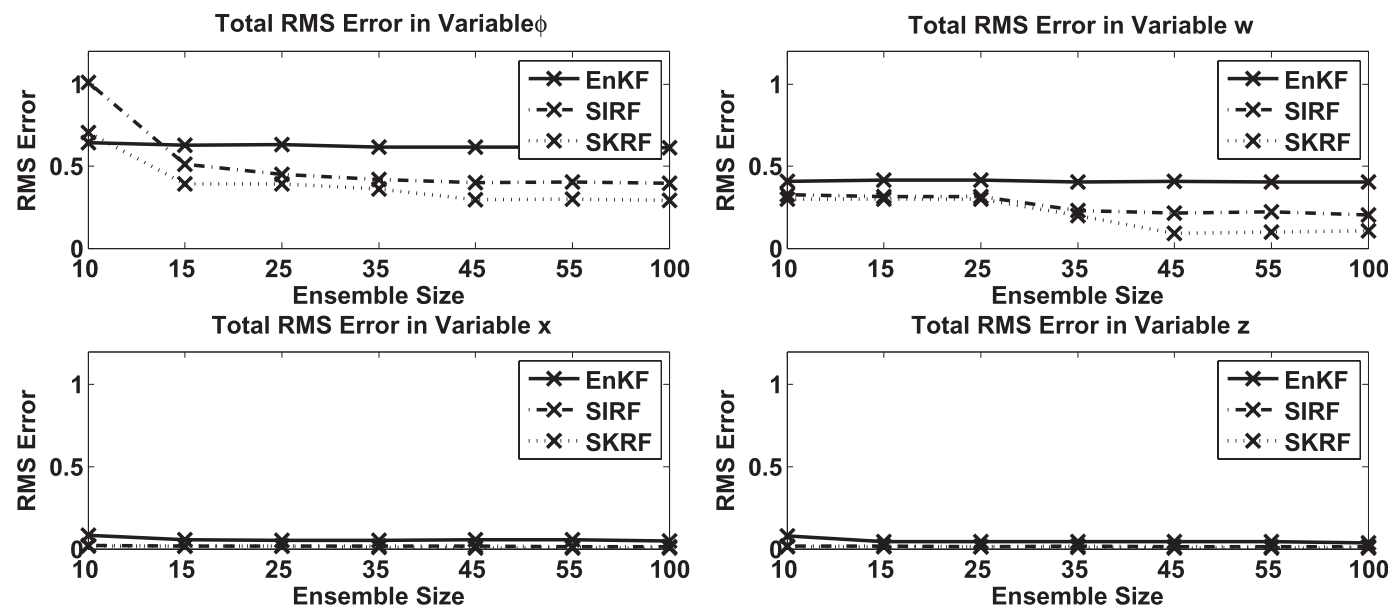

FIG. 7. Comparison of average error for all four variables in the "All modes" configuration of the model with observations taken at every eighth time step, showing errors for (top left) $\phi$, (top right) $w$, (bottom left) $x$, and (bottom right) $z$. The model was integrated forward for 3000 time steps to derive these error values. 


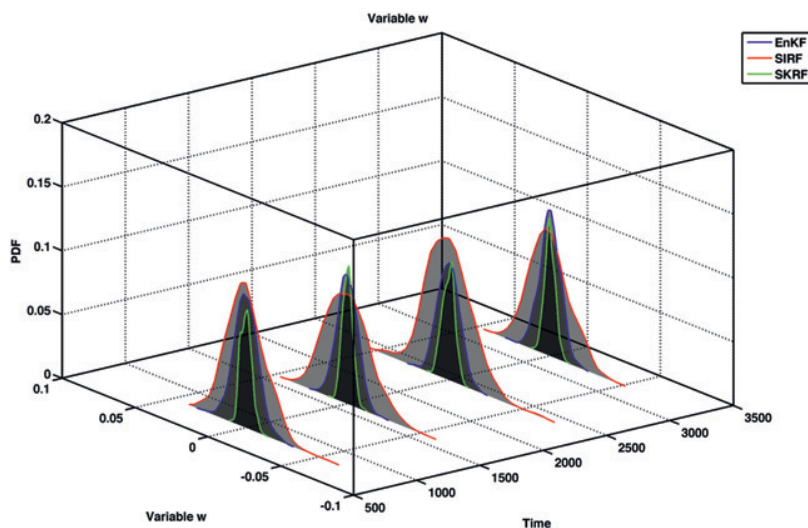

(a) Variable 2 Forecast

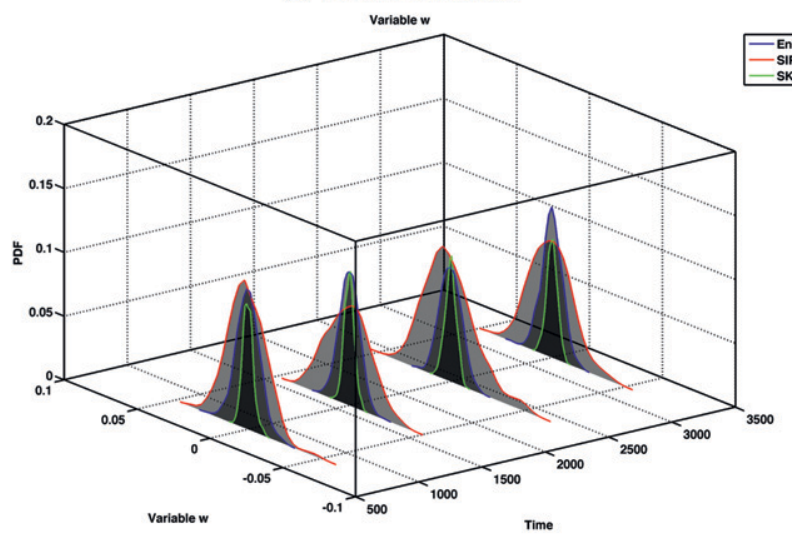

(c) Variable 2 Analysis

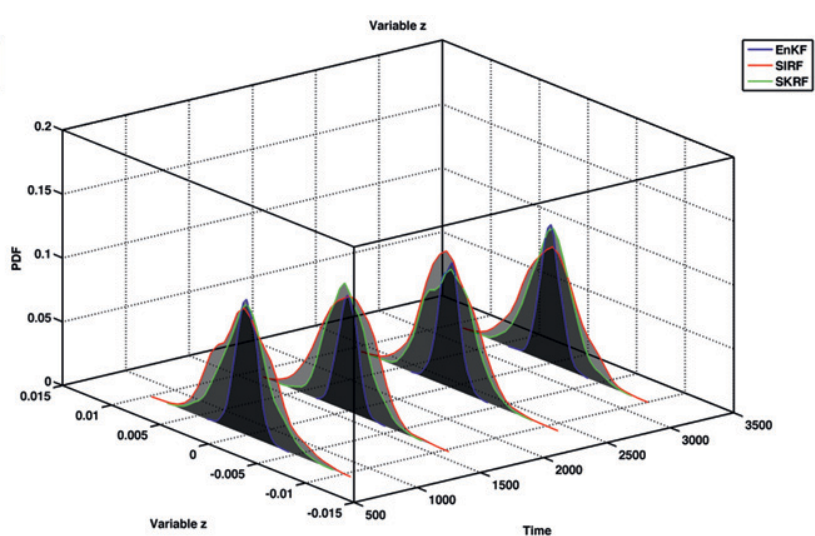

(b) Variable 4 Forecast

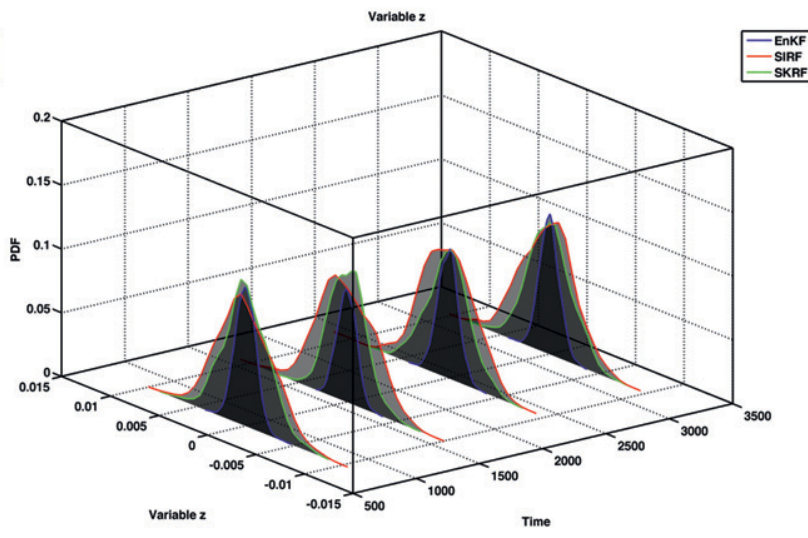

(d) Variable 4 Analysis

FIG. 8. All manifold: PDFs of forecast and analysis variables $w$ and $z$ for the "All modes" case estimated using a Gaussian mixture model are shown as a function of time. The filters were run with observations taken at every time step of the model run and the ensemble size of the filters was 1000 ensemble members.

in the observations, perhaps because of filtering or averaging of observations. In that case, only the component that is slaved to the slow mode can be controlled by observations, which requires the filter to simulate the balance relationships between slow and fast model variables.

Hence, the above experiments show that in estimating states with a weakly nonlinear model or a highly nonlinear model with interactions between the fast and slow variables in balance, both the nonlinear filters unequivocally give better estimates of the state variables than the EnKF, which is based on a Kalman correction.

\section{Discussion and conclusions}

Since its introduction five decades ago, Kalman filtering has been adapted as one of the most promising tools for data assimilation. The Kalman filter is an optimal linear filter. Hence, two different approaches are generally used for the implementation of this estimation technique to nonlinear models. The first approach consists of linearizing the model equations leading to the socalled extended Kalman filter. This approach has been shown either to be too prohibitive computationally or to have limitations in reduction in error of estimation for strongly nonlinear systems with simplified versions of the filter. Another approach is to use linear analysis-based Kalman filter for nonlinear estimation; this is based on the ensemble approach and the use of nonlinear Monte Carlo ensemble forecasting methods to represent estimation errors with an ensemble of state vectors. Until recently, these linearized filters have been shown to perform relatively well in state estimation of multidimensional nonlinear problems compared to other approximate methods such as optimal interpolation. The two main disadvantages that plague all Kalman filter-based approaches are that they do not produce the varianceminimizing estimate in the analysis step for nonlinear models and they initialize the Fokker-Planck-Kolmogorov equation with an ensemble that preserves only the first 


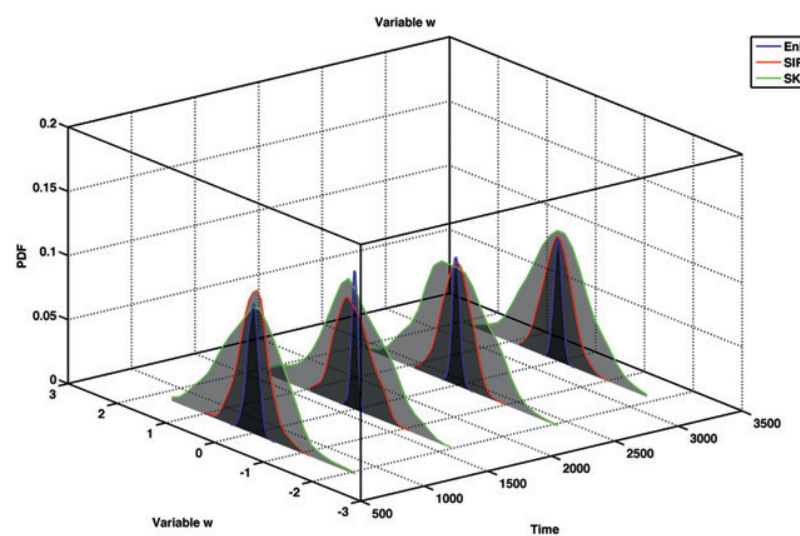

(a) Variable 2 Forecast

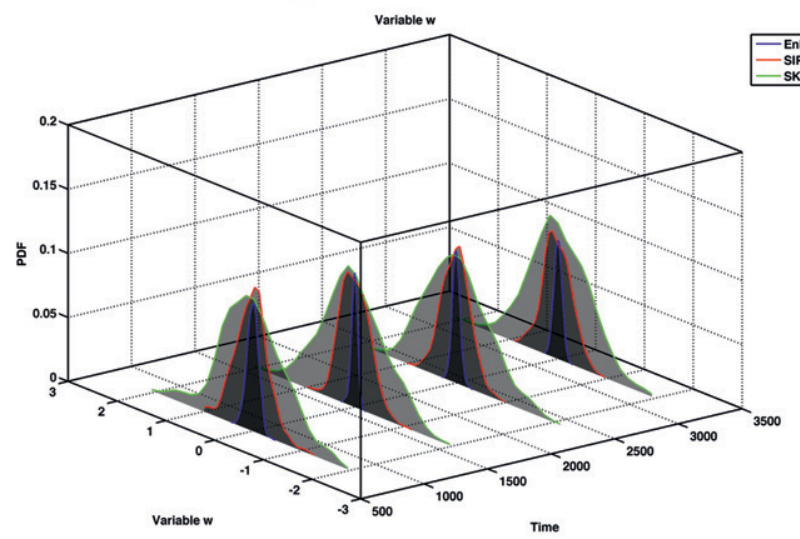

(c) Variable 2 Analysis

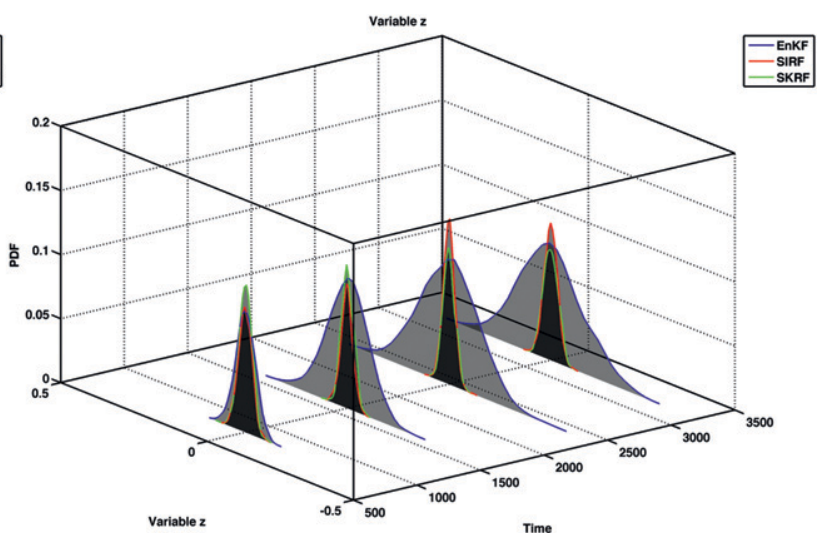

(b) Variable 4 Forecast

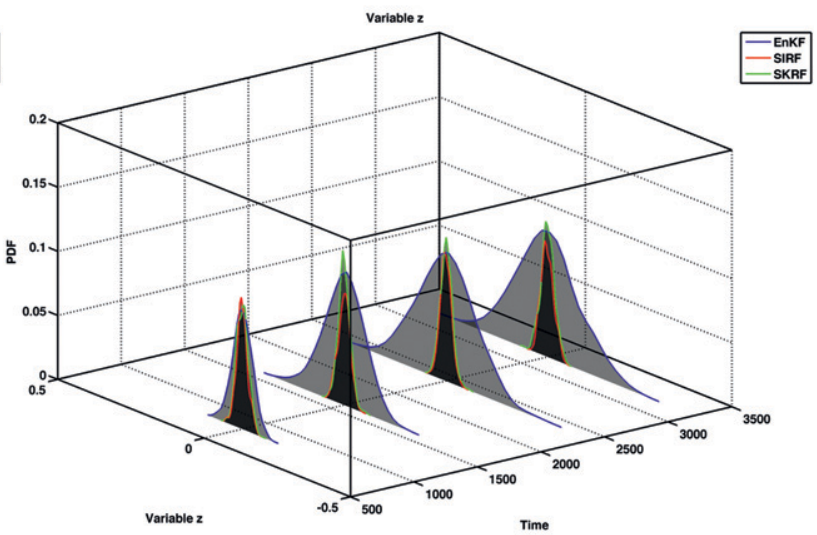

(d) Variable 4 Analysis

FIG. 9. All manifold: PDFs of forecast and analysis variables $w$ and $z$ for the "All modes" case estimated using a Gaussian mixture model are shown as a function of time. The filters were run with observations taken at every eighth time step of the model run and the ensemble size of the filters was 1000 ensemble members.

two moments of the analysis error statistics. It is also not completely understood how these filters influence the state estimation of different scales of dynamics.

Particle filters, on the other hand, are fully nonlinear in both model evolution and analysis steps (Doucet et al. 2001; Gordon et al. 1993). They use the full error statistics in filtering and hence minimize the true variance and not an assumed Gaussian variance. They are more suited for nonlinear estimation and nonlinear Monte Carlo ensemble forecasting of highly nonlinear processes such as ocean-atmosphere dynamics than linear-based Kalman filter. Yet, a fundamental problem with PFs is the so-called "curse of dimensionality", which is related to the fact that a relatively small number of model ensemble runs trying to estimate a large-dimensional system space is very unlikely to be close to the set of observations from this system (Snyder et al. 2008). More complicated particle filters have been proposed that can overcome the curse of dimensionality but they have not been used in geoscience applications very much (Van Leeuwen 2009). We have tested two such modified PFs, the SIRF and the SKRF, to understand how these filters influence the estimation of different scales of dynamics.

In this paper, we have compared the efficiency of the EnKF with the two flavors of nonlinear particle filters, the SIRF and the SKRF, for problems (i) where there exists a separation of time scales between relatively fast and slow motions, (ii) where the free fast motion is oscillatory, and (iii) where the evolution of the fast variables in the true state is slaved to that of the slow. These experiments showed that the SKRF, SIRF, and EnKF have quite different properties when it comes to preserving balance in the assimilated analysis. The PFs always outperformed the EnKF with any of the tested schemes of observation frequencies or modes of the model. This strongly suggests that a filter preserving the nonlinearity in the forward model tends to also preserve the balance in the model. 
Application of particle filters is attractive from the viewpoint that they use the full error statistics to integrate the Fokker-Planck-Kolmogorov equations of the system, unlike a Kalman filter, which uses only the first two moments in its integration. Hence, they are a truly variance-minimizing scheme. Numerical results from experiments with the exL86 model show that nonlinear filters behave much better than the ensemble Kalman filter methods with strongly nonlinear systems. The nonlinear filters also better preserve the dynamical balance of the system state resulting in more stable predictions in the slow and fast variables. Particle filters, especially SKRF, capture slaved modes better, implying that nonlinear jumps in dependent variables are simulated better. When the observation frequency and the number of variables observed are decreased, the nonlinear filters show a very clear improvement in performance compared to the linear analysis-based Kalman filter estimations for both the slow and fast variables in all configurations of the model. This is very important for atmospheric and oceanic data assimilation where only a small fraction of the system state is observed.

This study is intended as a complement to similar studies such as Neef et al. (2006) and studies involving larger, more complicated models, such as Mitchell et al. (2002). Key points of the balance problem and drawbacks of linear assumptions in estimation theory compared to nonlinear filters highlighted in this study add to the research in the still-evolving field of $4 \mathrm{D}$ data assimilation. This research can be extended to study the balance dynamics in more complicated models to understand the behavior of nonlinear filters in systems with strong nonlinearity.

Acknowledgments. Ibrahim Hoteit and Aneesh Subramanian gratefully acknowledge funding from ONR Grant N00014-08-1-0554. The views expressed herein are those of the authors and do not necessarily reflect the views of these agencies. Fruitful discussions with Lisa Neef are gratefully acknowledged. We thank the two referees for their careful reviews and important comments that significantly improved the manuscript.

\section{REFERENCES}

Anderson, B., and J. B. Moore, 1979: Optimal Filtering. Information and System Sciences Series, Prentice-Hall, $357 \mathrm{pp}$.

Anderson, J., 2001: An ensemble adjustment Kalman filter for data assimilation. Mon. Wea. Rev., 129, 2884-2903.

_- 2003: A local least squares framework for ensemble filtering. Mon. Wea. Rev., 131, 634-642.

Bennett, A., 2002: Inverse Modeling of the Ocean and Atmosphere. Cambridge University Press, 234 pp.

Burgers, G., P. Jan van Leeuwen, and G. Evensen, 1998: Analysis scheme in the ensemble Kalman filter. Mon. Wea. Rev., 126, 1719-1724.
Courtier, P., and O. Talagrand, 1990: Variational assimilation of meteorological observations with the direct and adjoint shallow-water equations. Tellus, 42, 531-549.

Dee, D., 1991: Simplification of the Kalman filter for meteorological data assimilation. Quart. J. Roy. Meteor. Soc., 117, 365384.

Doucet, A., S. Godsill, and C. Andrieu, 2000: On sequential Monte Carlo sampling methods for Bayesian filtering. Stat. Comput., 10, 197-208.

_ N. N. De Freitas, and N. Gordon, 2001: Sequential Monte Carlo Methods in Practice. Springer, 581 pp.

Evensen, G., 1994: Sequential data assimilation with a nonlinear quasi-geostrophic model using Monte Carlo methods to forecast error statistics. J. Geophys. Res., 99, 10 143-10 162.

_ 2003: The ensemble Kalman filter: Theoretical formulation and practical implementation. Ocean Dyn., 53, 343-367.

Gershgorin, B., and A. Majda, 2010: Filtering a nonlinear slow-fast system with strong fast forcing. Commun. Math. Sci., 8, 67-92.

Gordon, N., D. Salmond, and A. Smith, 1993: Novel approach to nonlinear/non-Gaussian Bayesian state estimation. IEE Proc., 140F, 107-113.

Hamill, T., and C. Snyder, 2000: A hybrid ensemble Kalman filter3D variational analysis scheme. Mon. Wea. Rev., 128, 2905-2919.

_ tering of background error covariance estimates in an ensemble Kalman filter. Mon. Wea. Rev., 129, 2776-2790.

Hoteit, I., B. Cornuelle, A. Köhl, and D. Stammer, 2005a: Treating strong adjoint sensitivities in tropical eddy-permitting variational data assimilation. Quart. J. Roy. Meteor. Soc., 131, 36593682.

— G. Korres, and G. Triantafyllou, 2005b: Comparison of extended and ensemble based Kalman filters with low and high resolution primitive equation ocean models. Nonlinear Processes Geophys., 12, 755-765.

—, D. Pham, G. Triantafyllou, and G. Korres, 2008: Particle Kalman filtering for data assimilation in meteorology and oceanography. Proc. Third WCRP Int. Conf. on Reanalysis. Tokyo, Japan, WCRP, 1-6.

Houtekamer, P., and H. Mitchell, 2005: Ensemble Kalman filtering. Quart. J. Roy. Meteor. Soc., 131, 3269-3289.

Jardak, M., I. Navon, and M. Zupanski, 2010: Comparison of sequential data assimilation methods for the KuramotoSivashinsky equation. Int. J. Numer. Methods Fluids, 62, 374-402.

Kalnay, E., H. Li, T. Miyoshi, S.-C. Yang, and J. Ballabrera-Poy, 2007: 4-D-Var or ensemble Kalman filter? Tellus, 59A, 758773.

Kepert, J. D., 2004: On ensemble representation of the observationerror covariance in the ensemble Kalman filter. Ocean Dyn., 54, $561-569$.

Kivman, G., 2003: Sequential parameter estimation for stochastic systems. Nonlinear Processes Geophys., 10, 253-259.

Le Dimet, F., and O. Talagrand, 1986: Variational algorithms for analysis and assimilation of meteorological observations: Theoretical aspects. Tellus, 38, 97-110.

Lewis, J. M., and J. Derber, 1985: The use of adjoint equations to solve a variational adjustment problem with advective constraints. Tellus, 37, 309-322.

Lorenc, A., 2003: The potential of the ensemble Kalman filter for NWP-A comparison with 4D-Var. Quart. J. Roy. Meteor. Soc., 129, 3183-3203.

Lorenz, E., 1986: On the existence of a slow manifold. J. Atmos. Sci., 43, 1547-1557. 
1991: Dimension of weather and climate attractors. Nature, 353, 241-244.

McLachlan, G., and D. Peel, 2000: Finite Mixture Models. John Wiley \& Sons, 419 pp.

Mitchell, H., and P. Houtekamer, 2000: An adaptive ensemble Kalman filter. Mon. Wea. Rev., 128, 416-433.

—,- , and G. Pellerin, 2002: Ensemble size, balance, and model-error representation in an ensemble Kalman filter. Mon. Wea. Rev., 130, 2791-2808.

Nakano, S., G. Ueno, and T. Higuchi, 2007: Merging particle filter for sequential data assimilation. Nonlinear Processes Geophys., 14, 395-408.

Neef, L. J., S. M. Polavarapu, and T. G. Shepherd, 2006: Fourdimensional data assimilation and balanced dynamics. J. Atmos. Sci., 63, 1840-1858.

$\longrightarrow, \ldots$, and 2009: A low-order model investigation of the analysis of gravity waves in the ensemble Kalman filter. J. Atmos. Sci., 66, 1717-1734.

Ott, E., and Coauthors, 2004: A local ensemble Kalman filter for atmospheric data assimilation. Tellus, 56A, 415-428.

Pham, D., 2001: Stochastic methods for sequential data assimilation in strongly nonlinear systems. Mon. Wea. Rev., 129, 1194 1207.

Polavarapu, S., M. Tanguay, and L. Fillion, 2000: Four-dimensional variational data assimilation with digital filter initialization. Mon. Wea. Rev., 128, 2491-2510.

$\mathrm{Pu}, \mathrm{Z}$., and J. Hacker, 2009: Ensemble-based Kalman filters in strongly nonlinear dynamics. Adv. Atmos. Sci., 26, 373-380.

Robinson, A. R., and P. Lermusiaux, 2004: Prediction systems with data assimilation for coupled ocean science and ocean acoustics. Theoretical and Computational Acoustics, D. A. Tolstoy et al., Eds., World Scientific, 325-342.
Saujani, S., and T. G. Shepherd, 2006: A unified theory of balance in the extratropics. J. Fluid Mech., 569, 447-464.

Snyder, C., T. Bengtsson, P. Bickel, and J. Anderson, 2008: Obstacles to high-dimensional particle filtering. Mon. Wea. Rev., 136, 4629-4640.

Song, H., I. Hoteit, B. Cornuelle, and A. Subramanian, 2010: An adaptive approach to mitigate background covariance limitations in the ensemble Kalman filter. Mon. Wea. Rev., 138, 2825-2845.

Szunyogh, I., E. Kostelich, G. Gyarmati, D. Patil, B. R. Hunt, E. Kalnay, E. Ott, and J. A. Yorke, 2005: Assessing a local ensemble Kalman filter: Perfect model experiments with the National Centers for Environmental Prediction global model. Tellus, 57A, 528-545.

Tippett, M., J. Anderson, C. H. Bishop, T. M. Hamill, and J. Whitaker, 2003: Ensemble square root filters. Mon. Wea. Rev., 131, 14851490.

Todling, R., 1999: Estimation theory and foundations of atmospheric data assimilation. DAO Office Note 1999-01, 187 pp.

— data assimilation based on the Kalman filter. Mon. Wea. Rev., 122, 2530-2557.

Van Leeuwen, P. J., 2009: Particle filtering in geophysical systems. Mon. Wea. Rev., 137, 4089-4114.

, 2010: Nonlinear data assimilation in geosciences: An extremely efficient particle filter. Quart. J. Roy. Meteor. Soc., 136, 1991-1999.

Wirosoetisno, D., and T. G. Shepherd, 2000: Averaging, slaving and balance dynamics in a simple atmospheric model. Physica $D, 141,37-53$.

Zou, Y., and R. Ghanem, 2005: A multiscale data assimilation with the ensemble Kalman filter. Multiscale Model. Simul., 3, 131150 . 